\title{
Research on the Diffraction Problem of Finite-length Anisotropic Impedance Split
}

Dun CAO

Computer \& Communication engineering Institute, Changsha University of Science \& Technology,

Changsha 410114, China 270161019@qq.com

\author{
Liling ZHEN
}

Computer \& Communication engineering Institute, Changsha University of Science \& Technology, Changsha 410114, China
Ming FU

Computer \& Communication engineering Institute, Changsha University of Science \& Technology, Changsha 410114, China

\begin{abstract}
Optimizing the channel and the radiation direction of the antenna is an important factor to improve the performance and quality of wireless communication systems. When it's rapidly developed in the field of communications, electrically large anisotropic impedance materials are used for the optimization of the antenna. However, how to calculate the high-frequency radiation, scattering and diffraction at high frequency of the electrically large anisotropic impedance conductor becomes a widespread problem . This paper uses the UTD, which belongs to the high-frequency analysis method, and the MEC together to solve the diffraction problem of finite anisotropic impedance split.In the end,I hope the methods could advance the follow-up research, and optimize the radiation performance of the entire base station antenna.
\end{abstract}

Keywords-UTD ; MEC ; Electrically Large Conductor ; Diffraction

\section{INTRODUCTION}

In recent years, many scholars were committing themselves to the diffraction problem of anisotropic impedance split. The UTD overcomes the shortcomings of the geometrical method of diffraction(GTD) and solve the continuous problem of electromagnetic waves in the shadow boundary transition region ${ }^{[1]}$. Then Russian scientists Lyalinov and Italian scientists Manara further learned the UTD solution of the anisotropic impedance split, and made it extending to a certain splitting angle $\mathrm{e}^{[2-4]}$, especially Manara's team used the perturbation method to calculate the UTD solution of anisotropic impedance split when deviated from the normal incidence in a small angle. However, the calculation of UTD could only use in the condition of the Keller cone,and could not calculate diffraction problem of finite length split.

The bringing out of the equivalent currents method extends UTD to any directions, and estimate the limited length of the split more accurately ${ }^{[5-9]}$. MEC use the equivalent currents as the source of the diffraction field, and the calculation of the radiation field only includes the line integral,so the calculation is relatively simple. Furthermore, the problem,that equivalent currents will become singular point in the shadow and reflection boundary transition zone, can be eliminated by subtracting the physical optics ${ }^{[10]}$. In conclusion, the combination of UTD and MEC can

This work is supported by Provincial Natural Science Foundation of Hunan under No. 11JJ3069;

Science Foundation of Hunan Province under Grant No. 2011SK3079. effectively solve the finite length diffraction in anisotropic impedance coated large target.

\section{DIFFRACTION RELATED TECHNOLOGIES}

Diffraction rays include diffraction rays, spire-rays of diffractions, surface diffraction rays ${ }^{[11]}$, which the edge and surface diffraction is more typical, this paper discusses the edge diffraction. Figure 1 is the edge of the physical model when a plane wave entering upon the anisotropic impedance split, Figure 2 shows the top view shown in Figure 1. Using the edge split as the $\mathrm{z}$-axis to create a cylindrical coordinate system, $n \pi$ express the size of outside split angle, $\overline{\bar{Z}}_{0}$ and $\overline{\bar{Z}}_{n}$ express the tensor surface impedance of anisotropic impedance split in $\phi=0$ and $\phi=n \pi$. Arbitrarily polarized plane wave along the direction of $\hat{i}=-\hat{x} \sin \beta_{0} \cos \phi_{0}-\hat{y} \sin \beta_{0} \sin \phi_{0}+\hat{z} \cos \beta_{0}$ irradiation to the split face of the anisotropic impedance, where $\beta_{0}$ is the angle of the incident wave vector and split edge, $\phi_{0}$ is the angle of wave vector and R split face. $P \equiv(\rho, \phi)$ is the observation point.

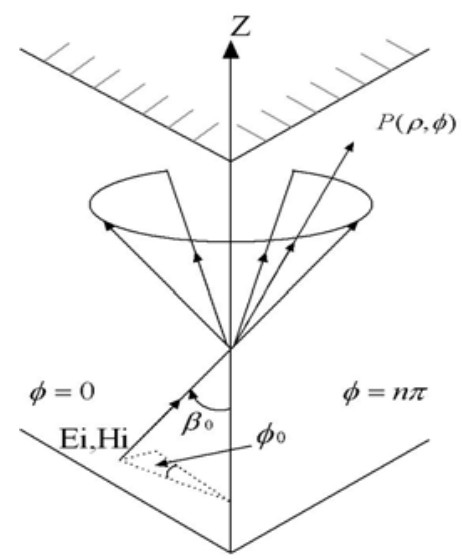

Fig. 1. The edge of the physical model when a plane wave entering upon the anisotropic impedance split 


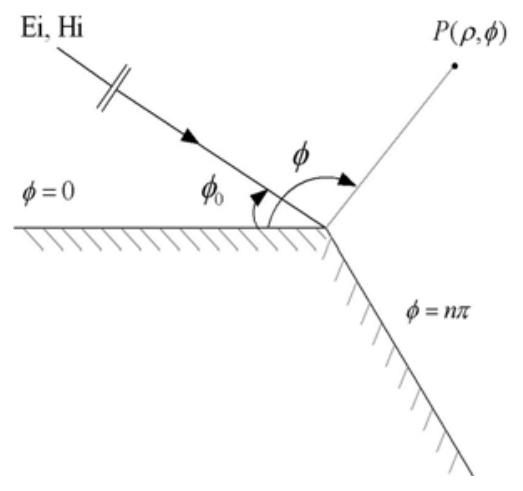

Fig. 2. Edge of the physical model top view

May have to field point $\mathrm{P}$ on the edge diffraction field as:

$$
E^{d}(P)=E^{i}(Q) \cdot \overline{\bar{D}} \sqrt{\rho_{c} / S \cdot\left(S+\rho_{c}\right)^{e^{-j k s}}}
$$

Where, $E^{i}(Q)$ is the incident field of
$Q, \rho_{c}=\frac{1}{\rho_{e}^{i}}-\hat{n}_{e}\left(\hat{s}^{\prime}-\hat{s}\right) / \rho_{e} \sin ^{2} \gamma$ is the difference between two main radius of the reflected ray wave.The parameter $S$ is the distance along the diffracted rays between diffraction point $Q$ to observation point $P$.A is the edge diffraction coefficient.

MEC is one of the practical way to solve diffraction problem,It overcomes the shortcomings of GTD which failure of caustics and can only calculate the diffraction field on the Keller cone.Estimating more accurate for the limited length of the split. The basis of the equivalent currents method is, when any far diffraction field of finite currents distribution through a radiation integral to sum, will be a limited result, by finding the appropriate distribution to avoid the UTD divergence ${ }^{[6]}$. However, the MEC theory has some shortcomings, In the algorithm, the diffraction coefficient in the shadow and reflection boundary transition zone will become singular point. According to this,we can add three coefficients of the physical optics to cancel out the singularity of diffraction coefficient, thus the diffraction coefficient remaining finite in reflect and shadow boundary transition region, and overcoming the shortcomings of several other diffraction calculation method ${ }^{[10]}$.

\section{A SOLUTION TO DIFFRACTION PROBLEM OF FINITE-LENGTH ANISOTROPIC IMPEDANCE SPLIT}

Uniform-geometrical theory of diffraction is developed on the basis of the geometrical theory of diffraction, It can only applies to calculate the diffraction of an infinitely long split, and can only be applied to the Keller cone; The method of equivalent currents extend UTD algorithm to an any direction, when any far diffraction field of finite currents distribution through a radiation integral to sum, will be a limited result. Both methods have their scope of application, so we consider them combined to calculate the diffraction problem of finite-length anisotropic impedance split.

In the MEC, the basic method is based on the assumption that there are line currents I and line magnetic currents $M$ at various points around the surface singularity (edge loops) and far field radiation integral in the form of summing them to represent the diffraction field ${ }^{[9]}$ :

$$
\vec{E}^{d}=-j k \int_{c^{\prime}}\left[Z I\left(\bar{r}^{\prime}\right) \hat{s} \times(\hat{s} \times \hat{t})+M\left(\bar{r}^{\prime}\right)(\hat{s} \times \hat{t})\right] G\left(\bar{r}^{\prime}, \bar{r}\right) d l
$$

Where, $\mathrm{k}$ is the number of incident wave; $\mathrm{Z}$ is the impedance of the medium; $\bar{r}$ and $\bar{r}^{\prime}$ is the position vector of the observation point and edge C; $G\left(\bar{r}, \bar{r}^{\prime}\right)=\exp (-j k s) / 4 \pi s$ is a three dimensional Green function. From this formula can be seen that the equivalent currents are fictional, because they depend on the scattering direction and the direction of incidence. The following figure shows the far-field Green's function diagram:

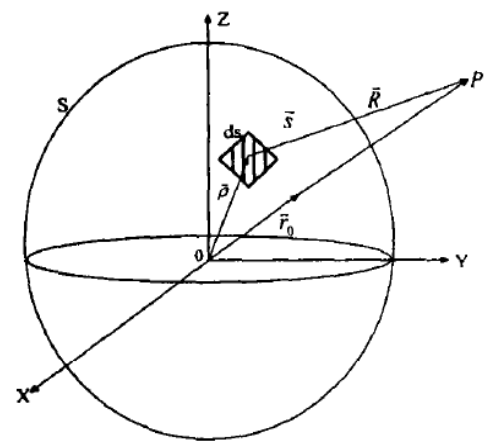

Fig. 3. Far-field Green's function diagram

In order to obtain the diffraction field, we must seek to qualify the line currents I and the line magnetic currents $M$ at first, which is the key. Of course, in this paper, we will combine the UTD algorithm, using the UTD's contribution at diffraction field,and a very strict conclusion after Mcihaeli study equivalent currents assumptions, we will not prove it, simply take this conclusion, so we can get the expression of equivalent currents as follows ${ }^{[8]}$ :

$$
\begin{aligned}
& I=I^{U-M}-I^{P O}=\frac{2 j}{K Z \sin ^{2} \beta_{t}} \vec{E}^{t} \cdot \hat{t} D_{22}+\frac{2 j}{K \sin ^{2} \beta_{t}} \vec{H}^{t} \cdot \hat{t} D_{21} \\
& M=M^{U-M}-M^{P O}=\frac{2 j}{K Y \sin \beta_{t} \sin \beta_{s}} \vec{H}^{t} \cdot \hat{t} D_{11}
\end{aligned}
$$

The above equation, we can solve the diffraction problem of finite-length anisotropic impedance split through solving the singular point problem of the equivalent currents at transition zone. In the method of equivalent currents,surface integral contains all near the edge of the currents (the uniform currents component and the non-uniform currents component), we will remove the 
uniform currents component, to eliminate the singularity.

Where $\mathrm{D}$ is the diffraction coefficient of equivalent currents, currents component is mapped to the diffraction coefficient, we can get the expression of being eliminated the singularity:

$$
\begin{aligned}
& D_{11}=D_{m}-D_{m}^{P O} \\
& D_{22}=D_{e}-D_{e}^{P O} \\
& D_{21}=D_{e m} \sin \beta_{i}-D_{e m}^{P O}
\end{aligned}
$$

Where,the integration of uniform-geometrical method of diffraction and method of equivalent currents is ${ }^{[12]}$ :

$$
\begin{aligned}
& {\left[\begin{array}{c}
E_{Z}^{d} \\
\eta_{0} H_{Z}^{d}
\end{array}\right]=\frac{e^{-j k \rho} e^{-j k z \cos \beta_{0}} e^{-j \frac{\pi}{4}}}{2 n \sqrt{2 \pi k_{t} \rho}}\left\{\left[\begin{array}{l}
D_{e}(\pi+n / 2-\phi) \\
D_{m}(\pi+n / 2-\phi)
\end{array}\right]\right.} \\
& \times\left(\cot \frac{\pi-\left(\phi-\phi_{0}\right)}{2 n} F\left\{k_{t} \rho\left[1+\cos \left(\phi-\phi_{0}\right)\right]\right\}\right. \\
& \left.-\cot \frac{\pi-\left(\phi+\phi_{0}\right)}{2 n} F\left\{k_{t} \rho\left[1+\cos \left(\phi-\phi_{0}\right)\right]\right\}\right) \\
& \left.+\left[\begin{array}{l}
D_{e}(-\pi+n \pi / 2-\phi) \\
D_{m}(-\pi+n \pi / 2-\phi)
\end{array}\right]\left(\cot \frac{\pi+\left(\phi-\phi_{0}\right)}{2 n}-\cot \frac{\pi+\left(\phi+\phi_{0}\right)}{2 n}\right)\right\}
\end{aligned}
$$

Where, $\Pi_{e, h}(\alpha)$ is the helper function of diffraction spectrum at Maliuzhinets methods. In the uniform-geometrical method of diffraction, the transition region on both sides of shadow boundary of geometrical optics will be effective,and it will degenerate into geometric diffraction theory formula automatically at outside.it is a good practical engineering computing .

Physical optics diffraction coefficient have been given in a lot of papers,Expression is shown below:

$$
\begin{gathered}
D_{m}^{P O}=-U^{+} \frac{\sin \varphi_{s}}{\cos a_{1}+\cos \varphi_{i}}-U^{-} \frac{\sin \left(n \pi-\varphi_{s}\right)}{\cos a_{2}+\cos \left(n \pi-\varphi_{i}\right)} \\
D_{e}^{P O}=-U^{+} \frac{\sin \varphi_{i}}{\cos a_{1}+\cos \varphi_{i}}-U^{-} \frac{\sin \left(n \pi-\varphi_{i}\right)}{\cos a_{2}+\cos \left(n \pi-\varphi_{i}\right)} \\
D_{e m}^{P O}=-U^{+}\left[\frac{Q \cos \rho_{s}}{\cos \phi_{1}+\cos p_{i}}-\cos \beta\right]+U\left[\frac{Q \operatorname{co}\left(n \pi-\varphi_{s}\right)}{\cos \phi_{2}+\operatorname{co}\left(n \pi-\varphi_{i}\right)}-\cos \beta_{i}\right]
\end{gathered}
$$

Using the result of (6) and (7) into the (5), we are able to get the diffraction coefficient value after eliminating the singularity, then get the expression of the electromagnetic flow. The final results substitute in the original style will be able to get the diffraction results of UTD under the MEC:

$$
\begin{aligned}
& \vec{E}^{d}=-j k \int_{c}\left\{Z\left[\frac{2 j}{K Z \sin ^{2} \beta_{t}} \vec{E}^{t} \cdot \hat{t} D_{22}+\frac{2 j}{K \sin ^{2} \beta_{t}} \vec{H}^{t} \cdot \hat{t} D_{21}\right] \hat{s} \times(\hat{s} \times \hat{t})\right. \\
& \left.+\frac{2 j}{K Y \sin \beta_{t} \sin \beta_{s}} \vec{H}^{t} \cdot \hat{t} D_{11}(\hat{s} \times \hat{t})\right\} G\left(\bar{r}^{\prime}, \bar{r}\right) d l
\end{aligned}
$$

The diffraction field is no longer confined to the Keller cone of a bus, it will extend UTD algorithm to an arbitrary direction, and get a finite value by means of integral. The above is the description of the diffraction problem-solving ideas of a finite-length anisotropic impedance split.By skillfully using The UTD and The MEC together,the problem can be properly solved.

\section{REFERENCES}

[1] Liu,P.,Tan,J.,Long,Y. Time Domain UTD-PO Solution for the Multiple Diffraction of Spherical Waves for UWB Signals.IEEE Transactions on Antennas and Propagation,2011, 59(4)

[2] Ghorbani, A.,Tajvidy, A.,Torabi, E.,Arablouei, R.. A new uniform theory of diffraction based model for multiple building diffraction in the presence of trees.Electromagnetics,2011, 31(1/4)

[3] Manara,G.P.Nepa, and G. Pelosi, and A.Vallecchi. EM scattering from anisotropic impedance half-plane, Electronics Letters, 2000, 36(6): 505-506

[4] Lyalinov, M. A., and N. Y. Zhu. Diffraction of a skewly incident plane wave by an anisotropic impedance wedge-A class of exactly solvable cases, Wave Motion, 1999, 30:275-288

[5] Jiang Xiangwen, Zhao Qijun, Xu Guohua. Calculation and Analysis of RCS of Helicopter Based on Panel Edge Method.Journal of nanjing university of aeronautics\&astronautics,2011, 43(3)

[6] Geng Fang-zhi,Lv Dan,Zhang Yong-xin,Deng Fa-sheng. Research of High Frequency Scattering Characteristic of Radar Cross Section on Shaping Stealth Targets[J]Electro-optic technology application, 2005,20(04)

[7] Sen Tian,Zhuo Li,Chang Qing Gu,Dan Ping Ding. Equivalent Electromagnetic Currents on Infinite Stratified Homogeneous Bi-anisotropic Media Backed by A PEC Layer. Applied Computational Electromagnetics Society journal,2011, 26(3)

[8] Mao Ying.Target edge scattering algorithm and its application[M].Northwestern Polytechnical University,2005,PP.6-14

[9] Qin Yalin. The high-frequency analysis and calculations of complex structure of multiple reflection and diffraction[M]. Northwestern Polytechnical University,2005,PP.15-24

[10] Xu Yun-xue, Gong Shu-xi. Treatment of false singularities in fringe components of equivalent edge currents[J]Journal of xidian university(natural science), 2007,34(02)

[11] Tan Yuanyang. Analysis of the scattering character of Airborne-antennas By UTD method(case of cylinder). Xi'an University of Electronic Science and Technology,2004,PP.3-10

[12] Li Ji,Yu Ding-feng,Yao Jing-jing,Zhu Guo-qiang.Scattering by wedge with anisotropic impedance faces at skew incidence: diffraction of surface waves[J]Systems engineering and electronics,2010,32(09) 